\title{
USP6 genetic rearrangements in cellular fibroma of tendon sheath
}

\author{
Jodi M Carter ${ }^{1}$, Xiaoke Wang ${ }^{1}$, Jie Dong ${ }^{1}$, Jennifer Westendorf ${ }^{2}$, Margaret M Chou ${ }^{3}$ and \\ Andre M Oliveira ${ }^{1}$ \\ ${ }^{1}$ Department of Laboratory Medicine and Pathology, Mayo Clinic, Rochester, MN, USA; ${ }^{2}$ Department of \\ Orthopedic Surgery, Mayo Clinic, Rochester, MN, USA and ${ }^{3}$ Department of Pathology and Laboratory \\ Medicine, Children's Hospital of Pennsylvania, Philadelphia, PA, USA
}

\begin{abstract}
Fibroma of tendon sheath is a benign (myo)fibroblastic neoplasm of the tenosynovial soft tissues, typically affecting the distal extremities. It is classically described as a paucicellular, densely collagenized tumor; however, cellular variants have been described. A subset of cellular fibromas of tendon sheath shares similar histological features with nodular fasciitis. As nodular fasciitis very frequently harbors rearrangement of ubiquitin-specific peptidase 6 (USP6), we hypothesized that cellular fibromas of tendon sheath with nodular fasciitis-like features may also contain USP6 rearrangements. Cases of fibroma of tendon sheath $(n=19)$, including cellular $(n=9)$ and classic $(n=10)$ variants, were evaluated for USP6 rearrangement by fluorescence in situ hybridization studies. A subset of cases was tested for MYH9 rearrangements and MYH9-USP6 and CDH11-USP6 fusion products. Classic fibroma of tendon sheath occurred in 5 males and 5 females (median age 67 years, range 23-77 years) as soft tissue masses of the hand $(n=4)$, finger $(n=3)$, forearm $(n=1)$ and foot $(n=2)$. Cellular fibroma of tendon sheath occurred in 5 males and 4 females in a younger age group (median age 32 years, range 12-46 years) as small soft tissue masses of the finger $(n=5)$, hand $(n=3)$ and wrist $(n=1)$. USP6 rearrangements were detected in 6/9 cellular fibromas of tendon sheath. Among cellular fibromas of tendon sheath with USP6 rearrangements, no MYH9 rearrangements were detected. By RT-PCR, neither the MYH9-USP6 or the CDH11-USP6 fusion products were detected in any case. Neither USP6 nor MYH9 rearrangement were detected in any classic fibroma of tendon sheath. We report for the first time the presence of USP6 rearrangements in a subset of cellular fibroma of tendon sheath. Based on the similar morphological and molecular genetic features, we suspect that a subset of cellular fibromas of tendon sheath are under-recognized examples of tenosynovial nodular fasciitis, driven by alternate USP6 fusion genes. Further investigation will delineate how these lesions should best be classified.
\end{abstract}

Modern Pathology (2016) 29, 865-869; doi:10.1038/modpathol.2016.83; published online 29 April 2016

Originally described by Geschickter and Copeland, ${ }^{1}$ fibroma of tendon sheath is a benign soft tissue tumor of the tenosynovial membranes, typically affecting the distal extremities. In 1979, in the largest clinicopathological series to date, Chung and Enzinger ${ }^{2}$ reported that fibromas of tendon sheath occur most commonly in middle-aged adults, with the finger, hand and wrist as the most common sites of involvement.

Classically, fibromas of tendon sheath are paucicellular neoplasms, composed of bland stellateto-spindled fibroblasts embedded in a dense fibro-

Correspondence: Dr JM Carter, MD, PhD, Department of Laboratory Medicine and Pathology, Mayo Clinic, 200 1st Street SW, Rochester, MN 55905, USA.

E-mail: carter.jodi@mayo.edu

Received 21 January 2016; revised 28 February 2016; accepted 18 March 2016; published online 29 April 2016 collagenous stroma. ${ }^{3}$ However, Chung and Enzinger ${ }^{2}$ noted a subset of fibromas of tendon sheath with moderate-to-high cellularity. These cellular fibromas of tendon sheath were composed, in part, of loosely formed fascicles of spindled-to-stellate fibroblasts with microcystic change and erythrocyte extravasation. Remarkably, they reported that the histological features were strikingly similar to nodular fasciitis.

Unlike fibroma of tendon sheath, nodular fasciitis is a common, self-limited soft tissue lesion that typically occurs in young adults and often presents as a rapidly growing mass with a predilection for the superficial soft tissues of the arm, trunk and head and neck. ${ }^{4,5}$ Typically, nodular fasciitis is composed of (myo)fibroblastic cells forming loosely arranged fascicles with a 'tissue culture-like' growth pattern. In 2011, Erickson-Johnson et $a l^{6}$ demonstrated that nodular fasciitis frequently contains a recurring reciprocal translocation involving the USP6 locus 
on chromosome 17. In their series, $92 \%$ of cases contained USP6 rearrangements wherein $65 \%$ of those were partnered with $M Y H 9$, a non-muscle myosin expressed by fibroblasts and other cell types. In 2013, Amary et $a 1^{7}$ reported an identical frequency of USP6 rearrangements in nodular fasciitis, with $92 \%$ of the cases in their series containing USP6 rearrangements. USP6 encodes a de-ubiquitinating enzyme, one member of a large family of proteases involved in intracellular protein trafficking and turnover. ${ }^{8}$ USP6 rearrangements also occur in a majority of aneurysmal bone cysts; however, the fusion partners differ from those seen in nodular fasciitis. ${ }^{9-14}$ As a subset of cellular fibromas of tendon sheath has overlapping histological features with nodular fasciitis, we hypothesized that cellular fibroma of tendon sheath may be a tenosynovial variant of nodular fasciitis and harbor USP6 genetic rearrangements. We tested a series of classic and cellular fibromas of tendon sheath for USP6 and MYH9 rearrangements and MYH9-USP6 and CDH11-USP6 fusion products.

\section{Materials and methods}

The study was approved by the Mayo Clinic Institutional Review Board. Our institutional and consultation archives were searched for cases coded as fibroma of tendon sheath, yielding 19 cases with available formalin-fixed, paraffin-embedded tissue blocks. All available slides were re-reviewed by two of the authors (JMC, AMO) and showed the histological features of classic or cellular variants of fibroma of tendon sheath as previously described. ${ }^{2}$ Designation as cellular variant of fibroma of tendon sheath required a minimum of $15 \%$ of the lesion to contain moderately to highly cellular areas. All cellular fibromas of tendon sheath also contained areas of classic fibroma of tendon sheath. Discrepant cases were resolved by consensus review.

\section{Fluorescence In Situ Hybridization (FISH)}

FISH studies for USP6 and MYH9 were performed in formalin-fixed, paraffin-embedded tissues using a break apart probe strategy and a probe set generated from commercially available BAC clones as previously described. ${ }^{6}$ A minimum of 200 nonoverlapping nuclei were scored and a rearranged locus was defined as one in which $>15 \%$ of lesional cells showed a splitting of the spectrum orange and green signals.

\section{RT-PCR}

RT-PCR for the MYH9-USP6 and CDH11-USP6 fusion products was performed on RNA retrieved from archival formalin-fixed, paraffin-embedded tissues as previously described. ${ }^{6}$ Briefly, $1 \mu \mathrm{g}$ of
RNA was reverse transcribed using the iScript Select cDNA Synthesis Kit using random primers per the manufacturer's instruction (Bio-Rad, Hercules, CA, USA). PCR was performed using specific primers as previously described. ${ }^{6}$

\section{High-Throughput Sequencing}

The Archer FusionPlex Sarcoma Panel was used to identify possible novel USP6 fusion transcripts using the vendor's recommended protocols (http:// archerdx.com/fusionplex-assays/sarcoma).

\section{Results}

The clinicopathological features of the fibromas of tendon sheath included in this study are summarized in Table 1. Following histological review, the 19 cases were classified as 10 classic and 9 cellular fibromas of tendon sheath. Classic fibromas of tendon sheath occurred predominantly in adults, median age 67 years (range 23-77 years), equally in males and females. They presented as small masses with an average size of $2.3 \mathrm{~cm}$ (range $0.7-6.5 \mathrm{~cm}$ ), involving the soft tissues of the hand $(n=4)$, finger $(n=3)$, arm $(n=1)$ and foot $(n=2)$. Cellular fibromas of tendon sheath occurred in a younger age group than the classic variant (median 32 years, range 1246 years), with an almost equal predilection for males and females. Similar to classic fibroma of tendon sheath, they presented as masses of the soft tissues of the finger $(n=5)$, hand $(n=3)$ and wrist $(n=1)$, with a mean size of $1.1 \mathrm{~cm}$ (range $0.5-1.6 \mathrm{~cm}$ ). Clinical follow-up data were available in 9 cases, including 3 cellular and 6 classic fibromas of tendon sheath, with a mean duration of 107 months (range

Table 1 Clinicopathological features and USP6 and MYH9 rearrangement status

\begin{tabular}{lclccc}
\hline $\begin{array}{l}\text { Variant of fibroma } \\
\text { of tendon sheath }\end{array}$ & Age/sex & Site & $\begin{array}{c}\text { Size } \\
\text { (cm) }\end{array}$ & USP6 & MYH9 \\
\hline Cellular & $24 / \mathrm{M}$ & Wrist & 1.6 & + & - \\
Cellular & $32 / \mathrm{F}$ & Hand & NA & + & - \\
Cellular & $33 / \mathrm{F}$ & Finger & NA & + & - \\
Cellular & $42 / \mathrm{F}$ & Finger & NA & + & - \\
Cellular & $12 / \mathrm{M}$ & Finger & NA & + & NA \\
Cellular & $46 / \mathrm{M}$ & Hand & NA & + & - \\
Cellular & $26 / \mathrm{F}$ & Finger & 0.5 & - & NA \\
Cellular & $31 / \mathrm{M}$ & Thumb & NA & - & NA \\
Cellular & $38 / \mathrm{M}$ & Hand & 1.1 & - & NA \\
Classic & $23 / \mathrm{F}$ & Finger & NA & - & NA \\
Classic & $43 / \mathrm{F}$ & Finger & 0.7 & - & NA \\
Classic & $52 / \mathrm{F}$ & Forearm & NA & - & - \\
Classic & $53 / \mathrm{M}$ & Foot & NA & - & - \\
Classic & $65 / \mathrm{F}$ & Hand & 1.2 & - & NA \\
Classic & $69 / \mathrm{M}$ & Hand & 1.2 & - & NA \\
Classic & $70 / \mathrm{M}$ & Wrist & 3.6 & - & NA \\
Classic & $71 / \mathrm{F}$ & Foot & 2 & - & NA \\
Classic & $71 / \mathrm{M}$ & Finger & 1 & - & NA \\
Classic & $77 / \mathrm{M}$ & Hand & 6.5 & - & NA \\
& & & & & \\
\hline
\end{tabular}



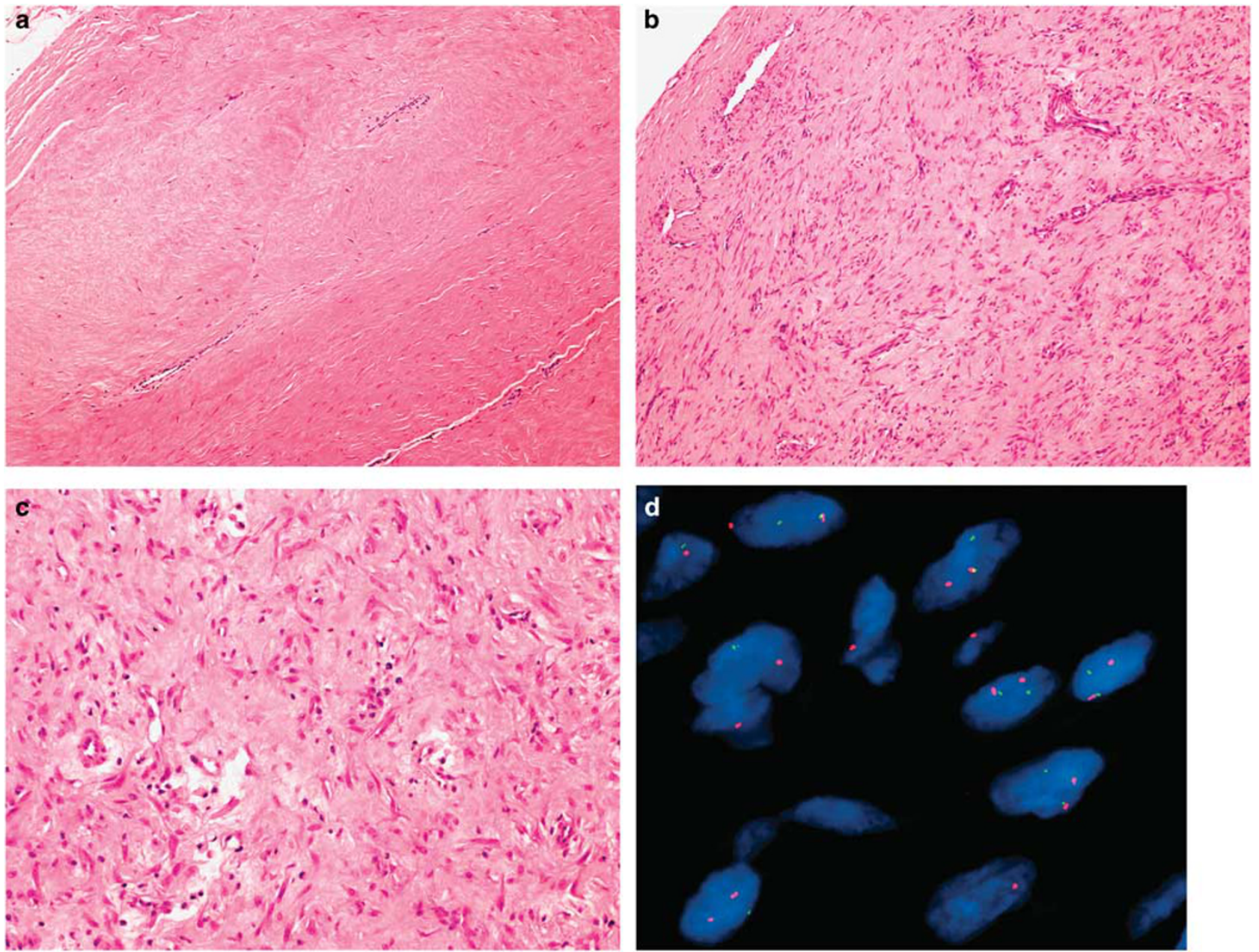

Figure 1 Classic and cellular variants of fibroma of tendon sheath. (a) Classic fibroma of tendon sheath is a paucicellular fibroblastic neoplasm with dense fibrocollagenous stroma. (b) Cellular fibroma of tendon sheath contains areas of increased cellularity composed of loosely formed fascicles of spindled-to-stellate fibroblasts. (c) Cellular fibroma of tendon sheath with areas of microcystic change and erythrocyte extravasation, histologically identical to those seen in nodular fasciitis. (d) Break apart USP6 FISH strategy in a cellular fibroma of tendon sheath. Separation of the green and orange signals indicates rearrangement of the USP6 locus.

60-192 months). All patients are currently alive with no evidence of disease.

The histological features of the classic and cellular fibromas of tendon sheath were similar to those previously described. ${ }^{2}$ Briefly, both classic and cellular fibromas of tendon sheath were well-circumscribed, lobular masses with no infiltrative features and displayed a characteristic slit-like, compressed vasculature at the lesional periphery. Classic fibromas of tendon sheath were composed of cytologically bland, spindled fibroblasts in a dense fibrocollagenous stroma (Figure 1a). In contrast, cellular fibromas of tendon sheath had variable amounts of a fascicle-forming, moderately cellular component (Figure 1b). The spindled-to-stellate cells showed mild pleomorphism with open chromatin and variably prominent nucleoli. Mitoses were identified in some cases but atypical mitoses were not seen. There was no significant cytological atypia. Within the cellular component, areas of microcystic change, extravasation of erythrocytes and scattered chronic inflammatory cells, reminiscent of nodular fasciitis, were present (Figure 1c). In these cases, the cellular areas merged into paucicellular, densely fibrotic areas with the histological features of classic fibroma of tendon sheath. All 19 cases, including the cellular and classic subsets, were tested for USP6 rearrangement by FISH as described in the Materials and Methods section. Among the classic fibromas of tendon sheath, none of the 10 cases had USP 6 rearrangements. Among the cellular fibromas of tendon sheath, 6 (of 9) cases showed rearrangement of the USP6 locus in both the cellular and 'classicappearing' components (Figure 1d). Although there was no difference in the clinical features between cellular fibromas of tendon sheath with and without USP6 rearrangements, one case lacking USP6 rearrangement did have a more distinctively storiform fascicular arrangement.

We further tested a subset $(n=7)$ of cases for MYH9 rearrangements, MYH9-USP6 and CDH11-USP6 fusion products, and possible novel USP6 fusion 
transcripts using a high-throughput sequencing approach. Cases included four USP6-rearranged cellular fibromas of tendon sheath, one cellular fibroma of tendon sheath without USP6 rearrangement and two classic fibromas of tendon sheath. None had MYH9 rearrangements or the MYH9-USP6 or CDH11-USP6 fusion products. After multiple trials, we could not identify a novel USP6 fusion transcript.

\section{Discussion}

Fibroma of tendon sheath is a rare benign soft tissue neoplasm, most commonly arising in the tenosynovial membranes of the distal extremities. ${ }^{3}$ As originally described by Geschickter and Copeland, ${ }^{1}$ fibromas of tendon sheath are typically paucicellular tumors, composed of scattered, stellate fibroblasts embedded within a fibrous stroma. Characteristically, they have 'slit-like' vessels at the lesional periphery. In 1979, Chung and Enzinger ${ }^{2}$ described a cellular variant of fibroma of tendon sheath composed primarily of fascicle-forming spindle cells often associated with extravasated erythrocytes, a mucoid matrix and scattered mononuclear cells. Although the authors noted the similarity of this subtype of cellular fibroma of tendon sheath to nodular fasciitis, they observed that the cellular areas invariably transitioned to the conventional hypocellular, collagenous areas of classic fibroma of tendon sheath. In addition to nodular fasciitis-like cellular fibroma of tendon sheath, they reported a cellular variant with dense fascicles of spindled cells similar to that seen in what was previously designated malignant fibrous histiocytoma. ${ }^{2}$

Subsequently, a handful of small series of fibroma of tendon sheath have confirmed the benign behavior of these lesions and the (myo)fibroblastic nature of the constituent cells, both by ultrastructural and immunohistochemical analysis. ${ }^{15-20}$ Although cases of cellular variants have been reported in almost all series, Pulitzer et $a l^{21}$ and Smith et $a l^{16}$ excluded nodular fasciitis-like examples from their respective series, arguing that such cases should be considered examples of tenosynovial nodular fasciitis rather than variants of fibroma of tendon sheath.

In our study, while six of the nine cellular fibromas of tendon sheath had USP6 rearrangements, no USP6 rearrangement was detected in any examples of classic fibroma of tendon sheath. Based on our data, we would also argue that at least a subset of nodular fasciitis-like cellular fibromas of tendon sheath represent under-recognized examples of tenosynovial nodular fasciitis. Although nodular fasciitis typically occurs in the subcutaneous soft tissues or rarely within skeletal muscle, it has been reported in unusual sites. ${ }^{22}$ Intra-articular nodular fasciitis has also been described and is reported to contain prominent stromal hyalinization. ${ }^{23}$ Although nodular fasciitis is typically composed of loosely arranged fascicles of (myo)fibroblastic cells with a 'tissue culture-like' growth pattern, Price et al ${ }^{24}$ were the first to recognize three variants of nodular fasciitis: myxoid, cellular, and fibrous subtypes. The conventional view is that these variants likely represent chronological stages of evolving nodular fasciitis. Accordingly, perhaps nodular fasciitis-like cellular fibroma of tendon sheath represents an evolving cellular and fibrous nodular fasciitis of the tenosynovial membranes. Interestingly, prior to this study, the reported median age of patients presenting with fibroma of tendon sheath was within the third or fourth decades; however, these series did not discriminate between classic and cellular subtypes. ${ }^{2,25}$ In our series, classic fibroma of tendon sheath occurred in a much older age group (median age 67 years) than cellular fibroma of tendon sheath (median age 32 years).

Classic fibroma of tendon sheath also appears to be genetically distinct from cellular fibroma of tendon sheath. Cytogenetic profiles have been reported in two cases of the classic type. In 1998, Dal Cin et al ${ }^{26}$ reported a $t(2 ; 11)(q 31-32 ; q 12)$ in a classic fibroma of tendon sheath of the hand in a 60-year-old woman. Similar translocations have been reported in desmoplastic fibroblastoma, raising the question of whether these entities are related. ${ }^{27-30}$ More recently, Nishio et $a l^{31}$ reported a complex karyotype, including a $t(9 ; 11)(p 24 ; q 13-14)$ translocation, among other abnormalities, in a classic fibroma of tendon sheath of the hand in a 38-year-old woman. Additional studies are required to clarify the genetics of these lesions.

The demonstration of USP6 rearrangements in nodular fasciitis was the first example of a selflimited lesion with a recurrent somatic gene fusion event. $^{6}$ USP6 (also known as TRE17 or TRE2) is located on chromosome 17p13 and encodes ubiquitinspecific peptidase 6 , a de-ubiquitinating enzyme involved in protein trafficking, inflammatory signaling and cell transformation. ${ }^{8}$ In nodular fasciitis, the most common chromosomal translocation breakpoints occur a $t(17 ; 22)(p 13: q 13)$ and result in a promoter swapping mechanism of the entire USP6-coding region with the promoter region of $M Y H 9$.

MYH9 is located on chromosome 22q12.3-q13 and encodes myosin heavy chain 9, a structural myosin classified within the non-muscle myosin class II family. ${ }^{32}$ MYH9 rearrangements are present in only $65 \%$ cases of nodular fasciitis, and alternative USP6 fusion partners exist. ${ }^{22}$ Presumably, similar to nodular fasciitis, USP6-rearranged cellular fibromas of tendon sheath have alternative, non-MYH9 fusion partners.

In summary, to date, the USP-induced family of neoplasia includes nodular fasciitis and aneurysmal bone cyst, including the subset of aneurysmal bone cyst previously described as giant cell reparative granuloma of the hands and feet. ${ }^{33,22}$ We report that cellular fibromas of tendon sheath frequently contain USP6 genetic rearrangements. USP6 rearrangements were not detected in classic fibromas of tendon sheath. Given the similar morphological and 
molecular genetic features to nodular fasciitis, we propose that cellular fibromas of tendon sheath with histological features of nodular fasciitis are likely a tenosynovial subset of nodular fasciitis. Additional study should delineate the frequency of USP6 rearrangements in these lesions and identify the USP6 genetic fusion partners.

\section{Acknowledgments}

We thank Michele Erickson-Johnson for assistance with data collection.

\section{Disclosure/conflict of interest}

The authors declare no conflict of interest.

\section{References}

1 Geschickter CF, Copeland MM. Tumors of Bone, Vol. 1, 3rd edn. J.B. Lippincott Co.: Philadelphia, PA, USA, 1949, pp 693-695.

2 Chung EB, Enzinger FM. Fibroma of tendon sheath. Cancer 1979;44:1945-1954.

3 Fletcher CD, Bridge JA, Hogendoorn PCW et al. WHO Classification of Tumours of Soft Tissue and Bone, Vol. 5, 4th edn. IARC: Lyon, France, 2013, pp 59-60.

4 Allen PW. Nodular fasciitis. Pathology 1972;4:9-26.

5 Konwaler BE, Keasbey L, Kaplan L. Subcutaneous pseudosarcomatous fibromatosis (fasciitis). Am J Clin Pathol 1955;25:241-252.

6 Erickson-Johnson MR, Chou MM, Evers BR et al. Nodular fasciitis: a novel model of transient neoplasia induced by MYH9-USP6 gene fusion. Lab Invest 2011;91:1427-1433.

7 Amary MF, Ye $\mathrm{H}$, Berisha $\mathrm{F}$ et al. Detection of USP6 gene rearrangement in nodular fasciitis: an important diagnostic tool. Virchows Arch 2013;463:97-98.

8 Oliveira AM, Chou MM. The TRE17/USP6 oncogene: a riddle wrapped in a mystery inside an enigma. Front Biosci (Schol Ed) 2012;4:321-334.

9 Oliveira AM, Hsi BL, Weremowicz S et al. USP6 (Tre2) fusion oncogenes in aneurysmal bone cyst. Cancer Res 2004;64:1920-1923.

10 Oliveira AM, Perez-Atayde AR, Dal Cin $\mathrm{P}$ et al. Aneurysmal bone cyst variant translocations upregulate USP6 transcription by promoter swapping with the ZNF9, COL1A1, TRAP150, and OMD genes. Oncogene 2005;24:3419-3426.

11 Oliveira AM, Chou MM, Perez-Atayde AR et al. Aneurysmal bone cyst: a neoplasm driven by upregulation of the USP6 oncogene. J Clin Oncol 2006;24:e1.

12 Ye Y, Pringle LM, Lau AW et al. TRE17/USP6 oncogene translocated in aneurysmal bone cyst induces matrix metalloproteinase production via activation of NF-kappaB. Oncogene 2010;29:3619-3629.

13 Lau AW, Pringle LM, Quick L et al. TRE17/ubiquitinspecific protease 6 (USP6) oncogene translocated in aneurysmal bone cyst blocks osteoblastic maturation via an autocrine mechanism involving bone morphogenetic protein dysregulation. J Biol Chem 2010;285:37111-37120.
14 Oliveira AM, Perez-Atayde AR, Inwards CY et al. USP6 and CDH11 oncogenes identify the neoplastic cell in primary aneurysmal bone cysts and are absent in so-called secondary aneurysmal bone cysts. Am J Pathol 2004;165:1773-1780.

15 Lundgren LG, Kindblom LG. Fibroma of tendon sheath. A light and electron-microscopic study of 6 cases. Acta Pathol Microbiol Immunol Scand A 1984;92:401-409.

16 Smith PS, Pieterse AS, McClure J. Fibroma of tendon sheath. J Clin Pathol 1982;35:842-848.

17 Hashimoto H, Tsuneyoshi M, Daimaru Y et al. Fibroma of tendon sheath: a tumor of myofibroblasts. A clinicopathologic study of 18 cases. Acta Pathol Jpn 1985;35: 1099-1107.

18 Jablokow VR, Kathuria S. Fibroma of tendon sheath. J Surg Oncol 1982;19:90-92.

19 Azzopardi JG, Tanda F, Salm R. Tenosynovial fibroma. Diagn Histopathol 1983;6:69-76.

20 Al-Qattan MM. Fibroma of tendon sheath of the hand: a series of 20 patients with 23 tumours. J Hand Surg Eur Vol 2014;39:300-305.

21 Pulitzer DR, Martin PC, Reed RJ. Fibroma of tendon sheath. A clinicopathologic study of 32 cases. Am J Surg Pathol 1989;13:472-479.

22 Oliveira AM, Chou MM. USP6-induced neoplasms: the biologic spectrum of aneurysmal bone cyst and nodular fasciitis. Hum Pathol 2014;45:1-11.

23 Hornick JL, Fletcher CD. Intraarticular nodular fasciitis-a rare lesion: clinicopathologic analysis of a series. Am J Surg Pathol 2006;30:237-241.

24 Price EB Jr, Silliphant WM, Shuman R. Nodular fasciitis: a clinicopathologic analysis of 65 cases. Am J Clin Pathol 1961;35:122-136.

25 Humphreys S, McKee PH, Fletcher CD. Fibroma of tendon sheath: a clinicopathologic study. J Cutan Pathol 1986;13:331-338.

26 Dal Cin P, Sciot R, De Smet L et al. Translocation 2;11 in a fibroma of tendon sheath. Histopathology 1998;32: 433-435.

27 Sciot R, Samson I, van den Berghe H et al. Collagenous fibroma (desmoplastic fibroblastoma): genetic link with fibroma of tendon sheath? Mod Pathol 1999;12:565-568.

28 Bernal K, Nelson M, Neff JR et al. Translocation $(2 ; 11)$ (q31;q12) is recurrent in collagenous fibroma (desmoplastic fibroblastoma). Cancer Genet Cytogenet 2004; 149:161-163.

29 Nishio J, Akiho S, Iwasaki H et al. Translocation $\mathrm{t}(2 ; 11)$ is characteristic of collagenous fibroma (desmoplastic fibroblastoma). Cancer Genet 2011;204:569-571.

30 Sakamoto A, Yamamoto H, Yoshida T et al. Desmoplastic fibroblastoma (collagenous fibroma) with a specific breakpoint of 11q12. Histopathology 2007;51:859-860.

31 Nishio J, Iwasaki H, Nagatomo $\mathrm{M}$ et al. Fibroma of tendon sheath with $11 \mathrm{q}$ rearrangements. Anticancer Res 2014;34:5159-5162.

32 D'Apolito M, Guarnieri V, Boncristiano M et al. Cloning of the murine non-muscle myosin heavy chain IIA gene ortholog of human MYH9 responsible for May-Hegglin, Sebastian, Fechtner, and Epstein syndromes. Gene 2002;286:215-222.

33 Agaram NP, LeLoarer FV, Zhang L et al. USP6 gene rearrangements occur preferentially in giant cell reparative granulomas of the hands and feet but not in gnathic location. Hum Pathol 2014;45:1147-1152. 